Paper presented at the 8th National Conference on Synchrotron Radiation

Instrumentation, August 23-26, 1993, Gaithersburg, MD.

Published in Nuclear Instruments and Methods in Physics Research A

347 (1994) 338-343.

\title{
Modeling Perfect Crystals in Transmission Geometry for Synchrotron Radiation Monochromator Design
}

\author{
M. Sánchez del Río and C. Ferrero \\ European Synchrotron Radiation Facility, BP 220, 30043 Grenoble-Cedex (France) \\ G-J Chen and F. Cerrina \\ Center for X-ray Lithography, 3731 Schneider Drive, Stoughton, Wisconsin 53589
}

We present here a modeling of Laue crystals in the framework of the ray-tracing program SHADOW [1]. In this program, the equations of the dynamical theory of $x$-ray diffraction have been coded to allow calculations of the diffracted intensity distributions. For thin and weakly absorbing crystal these profiles exhibit strong oscillations as consequence of the Pendellösung effect. These patterns produce an also oscillating value of the integrated intensity as a function of photon energy. The thickness of the crystal can be adjusted in order to optimize its optical performance for a given energy range. We analyze different examples of thickness optimization for such crystals. The performance of a Laue-Bragg monochromator is then compared to that of a standard Bragg-Bragg monochromator for a beamline configuration.

\section{Introduction}

Perfect crystals in transmission or Laue geometry are currently being considered as an interesting alternative to the more traditional Bragg-type monochromators commonly used at synchrotron radiation x-ray sources. Thin perfect crystals $(10-200 \mu \mathrm{m})$ of silicon or diamond diffracting photons in the hard $x$-ray range are able to produce an intense diffracted beam without involving high heat load. Such thin crystals would thus be extremely useful at third generation synchrotron radiation sources, where a large amount of power reaches the first optical element. In the case of the Laue crystals, almost all the beam is transmitted and the absorbed power is about one order of magnitude smaller than in the usual thick Bragg crystals. This, together with other factors, makes these crystals suitable for many purposes. For example, one of their interesting properties is that they can have a beneficial effect on beam focusing.

\section{Diffraction profile of a perfect crystal in Laue configuration}

The diffraction profile of a plane parallel perfect crystal wafer in transmission or Laue geometry can be calculated from the dynamical theory of x-ray diffraction as [2]:

$\mathrm{R}\left({ }_{\theta}\right) \equiv \frac{1}{|\mathrm{~b}|} \frac{\mathrm{I}_{\mathrm{H}}}{\mathrm{I}_{\mathrm{O}}}=\frac{1}{|\mathrm{~b}|}\left|\frac{\mathrm{x}_{1} \mathrm{x}_{2}\left(\mathrm{c}_{1}-\mathrm{c}_{2}\right)}{\mathrm{x}_{2}-\mathrm{x}_{1}}\right| 2$ 
where $\mathrm{I}_{\mathrm{H}}$ is the intensity of the external diffracted wave, $\mathrm{I} \quad \mathrm{o}$ is the intensity of the incident wave,

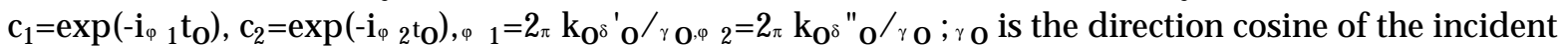
wave, $t_{O}$ is the crystal thickness and the other quantities are defined as:

$\delta_{\delta}^{\prime}{ }^{\prime} \mathrm{o}=\frac{1}{2}\left(\Psi \mathrm{o}-\mathrm{Z}_{ \pm} \sqrt{\left.\mathrm{q} \mathrm{P}^{2}+\mathrm{Z}^{2}\right)}\right.$

$\mathrm{x}_{1}=\frac{-\mathrm{z} \sqrt{\mathrm{qP}^{2}+\mathrm{z}^{2}}}{\mathrm{P}_{\Psi_{\mathrm{H}}}}$

$\mathrm{z}=\frac{1-\mathrm{b}}{2} \Psi_{\mathrm{o}}+\frac{\mathrm{b}}{2}$

with the following abbreviations: $\mathrm{q}=\mathrm{b}_{\Psi} \mathrm{H}^{\Psi} \mathrm{H}_{\mathrm{H}} \alpha=\left(1 /\left|\mathrm{k}_{\mathrm{O}}\right|^{2}\right)\left(\left|\mathrm{B}_{\mathrm{H}}\right|^{2}+2 \mathrm{k}_{\mathrm{H}} \cdot \mathrm{B}_{\mathrm{H}}\right) \approx 2\left({ }_{\theta}-\theta_{\mathrm{B}}\right) \sin { }_{\theta} \mathrm{B}, \mathrm{k}_{\mathrm{O}}$ is the wave vector of the incident wave in vacuum, $B \quad H_{H}=(-1 / d) u_{B}$ is the reciprocal lattice vector corresponding to the diffracting planes, $\quad \mathrm{u}_{\mathrm{B}}$ is the unit vector normal to the diffracted planes characterized by the Miller indices $(h, k, l),{ }_{\theta}$ is the rocking angle and ${ }_{{ }_{B}}$ is the kinematical Bragg angle. ${ }^{\Psi} \mathrm{H},{ }^{\Psi} \mathrm{H}$ and ${ }_{\mathrm{O}} \mathrm{O}$ are the Fourier components of the electrical susceptibility, $\mathrm{b}$ is the asymmetry factor defined as $b=\gamma_{0} / \gamma_{H}$, where $\gamma_{H}$ is the direction cosine of the diffracted wave; $\left|\quad k_{O}\right|=1 / \lambda_{, \lambda}$ is the photon wavelength in vacuum and $P$ is the polarization factor equal to 1 for the perpendicular polarization $(\sigma)$ and $\left|\cos 2_{\theta_{\mathrm{B}}}\right|$ for the parallel $\left(_{\pi}\right)$.

Eq 1 can be rewritten as:

$\mathrm{R}\left({ }_{\theta}\right)=\frac{\mathrm{b}\left|\mathrm{P}_{\psi} \mathrm{H}^{2}\right|^{2}}{\left|\mathrm{qP}^{2}+\mathrm{z}^{2}\right|} \exp \left(-_{-\mu} \mathrm{t}\right)\left[\sin ^{2}(\mathrm{av})+\sinh ^{2}(\mathrm{aw})\right]$

where:

$$
\mathrm{v}+\mathrm{iw} \equiv \sqrt{\mathrm{qP}} \mathrm{P}^{2}+\mathrm{z}^{2} \quad \mathrm{a} \equiv \pi \mathrm{k}_{\mathrm{O}} \mathrm{t}_{\mathrm{O}} / \gamma_{\mathrm{O}} \quad \mathrm{t} \equiv \frac{\mathrm{t}_{\mathrm{o}}}{2}\left(\frac{1}{\gamma_{\mathrm{O}}}+\frac{1}{\gamma_{\mathrm{H}}}\right)
$$

where ${ }{ }_{\mathrm{O}}$ is the linear absorption coefficient.

From this equation it is easy to identify that the shape of the diffraction profile is basically a Lorentzian $\frac{\mathrm{b}\left|\mathrm{P}_{\Psi}{ }_{\mathrm{H}}\right|^{2}}{\left|\mathrm{qP}^{2}+\mathrm{z}^{2}\right|}$ modified by the absorption present in the terms exp(- $\left.{ }^{\mu} \mathrm{o}\right)$ and $\sinh ^{2}(\mathrm{aw})$. In case of negligible absorption the two absorption terms become 1 and 0 , respectively. There is also an oscillatory term $\sin ^{2}$ (av) due to the Pendellösung effect. The oscillatory term superposes to the mean profile giving fringes in the total profile. The Pendellösung effect [3] is an interference effect of the wave field inside the crystal, which forms standing waves. The Pendellösung effect manifests itself in intensity variations of the diffracted intensity as a function of the rocking angle in the case of thin absorbing crystals, as in our case. It also manifests itself in the generation of interference fringes in the diffraction topographs of wedge-shaped parts of a perfect crystal [4].The oscillatory behavior is highly dependent on the crystal thickness, and its frequency increases with increasing thickness. In the limit of the thick crystal approximation, $\sin ^{2}(\mathrm{av})$ takes the mean value of $1 / 2$.

The SHADOW utility BRAGG can calculate these diffraction profiles and may be used as a standalone code for a first estimation of the crystal behavior. In Fig 1 we show an example of a calculation of this type, and highlight the contribution of the different partial terms to the total diffraction pattern. 


\section{Thickness optimization of a Laue crystal}

The "best" Laue crystal for monochromator applications delivers ideally the maximum diffracted light. Therefore a crystal ought to be chosen sufficiently thick to include many atomic layers in order to produce an intense diffraction; however it must be thin enough to avoid excessive absorption of the transmitted beam. In the intermediate interval, the crystal diffraction profiles will always present a strong Pendellösung effect. As it has already been stated, the oscillatory behavior of the diffraction profiles is strongly dependent on the crystal thickness and, additionally, on the polarization of the incident light. In the Laue case, the shape of diffracted profiles is very sensitive to the value of the polarization term, and may result in strong change of profiles as can be seen in Fig. 2 for $\sigma$ and, respectively, « polarization. Strong changes in the diffraction profiles can also be obtained by slightly changing the crystal thickness [5]. Thus, an appropriate choice of the crystal thickness is important as to have the maximum output in the selected energy for the planned experiment.

Furthermore, in the Laue case, the Pendellösung effect causes variations in the integrated intensity which is defined as:

$\mathrm{R}_{\mathrm{i}}=\int_{-\infty}^{\infty} \mathrm{R}(\theta) \mathrm{d}_{\theta}$

If one plots the integrated intensity as a function of the photon energy for different crystal thicknesses it is possible to select the optimum thickness for the desired energy range. In general, the integrated intensity of a Laue crystal is lower than that of a corresponding Bragg crystal. The ratio between the Laue and the Bragg integrated intensity can be calculated analytically for few limiting cases [2] and tends to the value 0.5 when thick crystals with no absorption are considered. Numerical calculations of the integrated intensity versus the crystal thickness and photon energy are shown for some crystals in Fig. 3 .

\section{Ray-tracing in a Laue crystal}

In order to calculate the output direction of each individual ray arriving onto the crystal, two assumptions are made. The first one is that the scattering is to be of elastic type, from conservation of the modulus of the wave-vector in the diffraction process:

$$
\left|\mathrm{k}_{\mathrm{H}}\right|=\left|\mathrm{k}_{\mathrm{O}}\right|
$$

The second assumption requires the continuity of the tangential components of the wavevectors. A straightforward consequence is the conservation of the area in the phase space diagram during, before and after the diffraction event. This can be seen as an implication of the Liouville theorem and has already been extensively discussed in relation with the ray tracing method [6], and is summarized in the next paragraph.

If an incident ray described by its wavevector $\quad k_{O}$ arrives on the crystal surface, it is diffracted producing an outgoing ray with wave-vector $\mathrm{k}_{\mathrm{H}}$. The crystal surface is defined locally by its normal $\mathrm{u}_{\mathrm{s}}$.

The continuity of the of tangential components of the wave vectors through the surface implies the conservation of the parallel components of wave vectors in the Laue equation:

$$
\mathrm{k}_{\mathrm{H}}||=\mathrm{k}_{\mathrm{O}}\left|+\mathrm{B}_{\mathrm{H}}\right| \mid
$$

The Laue equation $\mathrm{k}_{\mathrm{H}}=\mathrm{k}_{\mathrm{O}}+\mathrm{B}_{\mathrm{H}}$ is satisfied only if the ray falls on the crystal meeting exactly the Bragg condition corrected for refraction, which indicates the direction of maximum diffracted intensity. The normal components of the wave vectots in the Laue equation are not conserved to allow elastic scattering.

Eqs. 8 and 9 permit us to calculate the outlet direction of the incident rays. This is done by SHADOW for each individual ray of the incident beam. In the present version, the path followed by the ray 
inside the crystal is neglected. This approximation is reasonable when the distances source-crystal and crystal-image are much larger than the crystal thickness.

\section{Focusing properties of flat Laue crystals}

If the incident ray is contained in the plane formed by the vectors $u_{\mathrm{B}}$ and $\mathrm{u}_{\mathrm{S}}, \mathrm{Eq} .9$ can be written as a function of the incident ${ }_{\theta} \mathrm{O}$ and exit ${ }_{{ } \mathrm{H}}$ angles as follows (see fig 4):

$\sin { }_{\theta_{\mathrm{H}}}=\sin _{\theta_{\mathrm{O}}}-(\lambda / \mathrm{d}) \sin \alpha$

and its derivative

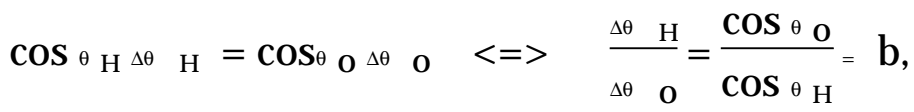

where $\mathrm{b}$ is the asymmetry factor introduced by Eq. 1, which is positive in the Laue case and negative in the Bragg case. As a consequence of the Liouville theorem applied to the photon beam propagation, a change in divergence induced by an asymmetrically cut crystal must be accompanied by a compensating change in the transverse cross section $S$ of the beam:

${ }_{\Delta} \mathrm{S}_{\mathrm{H} \Delta \theta}{ }_{\mathrm{H}}={ }_{\Delta} \mathrm{S}_{\mathrm{O}^{\Delta \theta}} \quad \mathrm{o} \quad \Leftrightarrow \quad \mathrm{b}=\frac{{ }_{\Delta} \mathrm{S}_{\mathrm{O}}}{{ }_{\Delta} \mathrm{S}_{\mathrm{H}}}$

Thus, an asymmetric flat Laue crystal is a focusing device because it induces a change in the divergence of the diffracted beam. For a monochromatic divergent incident beam, the diffracted beam is divergent as a consequence of the ray tracing Eqs. 8 and 9 (Fig 4a), and the angular acceptance of the crystal is given by its diffraction profile. On the contrary, for an incident polychromatic beam, for which divergence is larger than the diffraction profile width, the diffracted beam is switched to be convergent, as a result of the selective "reflection" on the Bragg planes as a function of the Bragg angle (Fig. 4b). Therefore, a polychromatic beam after the diffraction will converge until a best focus position or pseudo-focus which can be found at the distance $s_{2}$ from the Laue crystal:

$\mathrm{S}_{2}=\frac{\Delta \theta \mathrm{O}}{\Delta \theta \mathrm{H}} \mathrm{S}_{1}=\frac{1}{\mathrm{~b}} \mathrm{~S}_{1}$

where $s_{1}$ is the source-crystal distance. The size of the focus is not simply depending of the source size, and cannot go below the monochromatic case. The change of the ray direction in diffraction is not specular but is a typically dispersive process, with the only exception of the symmetric Bragg case. That means that rays with different wavelengths are diffracted following different directions. Therefore the spot size is also a function of the energy band pass of the Laue crystal itself and, if present, crystals or slits placed upstream or downstream of it. In cases like that, ray tracing calculations appear to be a very effective and powerful tool for calculating the focusing and energetic characteristics of monochromators based upon Laue crystals. The effect of the beam size dependence on energy resolution is illustrated in Fig. 5. The focusing effect of an asymmetric diamond crystal under an undulator beam has recently been experimentally measured at ESRF with excellent agreement with calculations done with SHADOW [7]

\section{Application example: comparison between a Bragg-Bragg and Laue-Bragg monochromator}

The use of a Laue Bragg monochromator can be extremely advantageous in the case of high thermal loads, because the beam is almost entirely transmitted, and only a small part is absorbed. Therefore they can have many applications for the third generation synchrotron radiation facilities, such as 
ESRF or APS. In fact, monochromators of this type are currently being designed, built and tested at ESRF [8].

Another advantage of monochromators using Laue crystals is the possibility of using two beams: the diffracted monochromatic one and the transmitted white one. The Laue crystal acts as a hard x-ray beam splitter [9]. The mechanical construction can be simplified cutting a "L"-shaped Laue-Bragg monochromator out of one crystal block. Rotating this device around the "L" vertex, one could scan different wavelengths conserving a fixed exit beam [8]. Another possibility would be to fully utilize its focusing properties, avoiding the use of other focusing devices or bending the crystal itself. Disadvantages include the difficulty of cooling a very thin crystal, along with the difficulty of shaping parallel crystal wafers with the required thickness and finally the lower diffracted intensity as compared with the Bragg crystal.

Fig. 6 shows the ray tracing results of a comparison of the performance of a standard double crystal Bragg monochromator and of a Laue-Bragg monochromator. As a consequence, one can state that the total intensity delivered by a Laue-Bragg monochromator is slightly less than the Bragg-Bragg case, but if one may exploit the focusing properties of the Laue crystal, the brightness or intensity per unit of area at the spot position may be more favorable for the Laue-Bragg monochromators.

\section{Conclusions}

It has been shown that the computer package SHADOW can be extremely useful to calculate the performance of synchrotron radiation monochromators designed with flat Laue crystals. Some characteristics of these crystals together with their applications have been presented to illustrate the capabilities of the code. Further work is in progress to extend the described method to the case of bent and ground-and-bent Laue crystals.

Acknowledgments

We wish to acknowledge the helpful discussions with J. Härtwig, M. Wulff, and P. Suortti, who clarified some aspects of diffraction in Laue crystals. We specially thank J. Härtwig for the critical review of the manuscript.

\section{References}

[1] C. Welnak, G-J Chen and F. Cerrina, These proceedings.

[2] W.H. Zachariasen, "Theory of X-ray Diffraction in Crystals" (Dover, New York, 1945)., pag 120

[3] P.P. Ewald, Ann d. Phys. (Leipzig.) 49 (1916) 117.

[4] N. Kato and A.R. Lang, Acta Crystallogr, 12 (1959) 787.

[5] T. Ishikawa, K. Hirano and S. Kikuta, Nucl. Intr. and Meth. A308 (1991) 356

[6] M. Sánchez del Río and F. Cerrina, Rev. Sci. Instr. 63, 1 (1992) 938

[7] J. Als-Nielsen, A. Freund, G. Grübel, J. Linderholm, M. Nielsen, M. Sánchez del Río and J.P.F.

Sellschop, To be submitted to Nucl. Instr. and Meth.

[8] M. Wulff and F. Sette, private communications.

[9] G. Grübel and J. Als-Nielsen. "The Troika beamline" ESRF annual report (1992) 127.

[10] M. Wulff. ESRF annual report (1992) 106. 


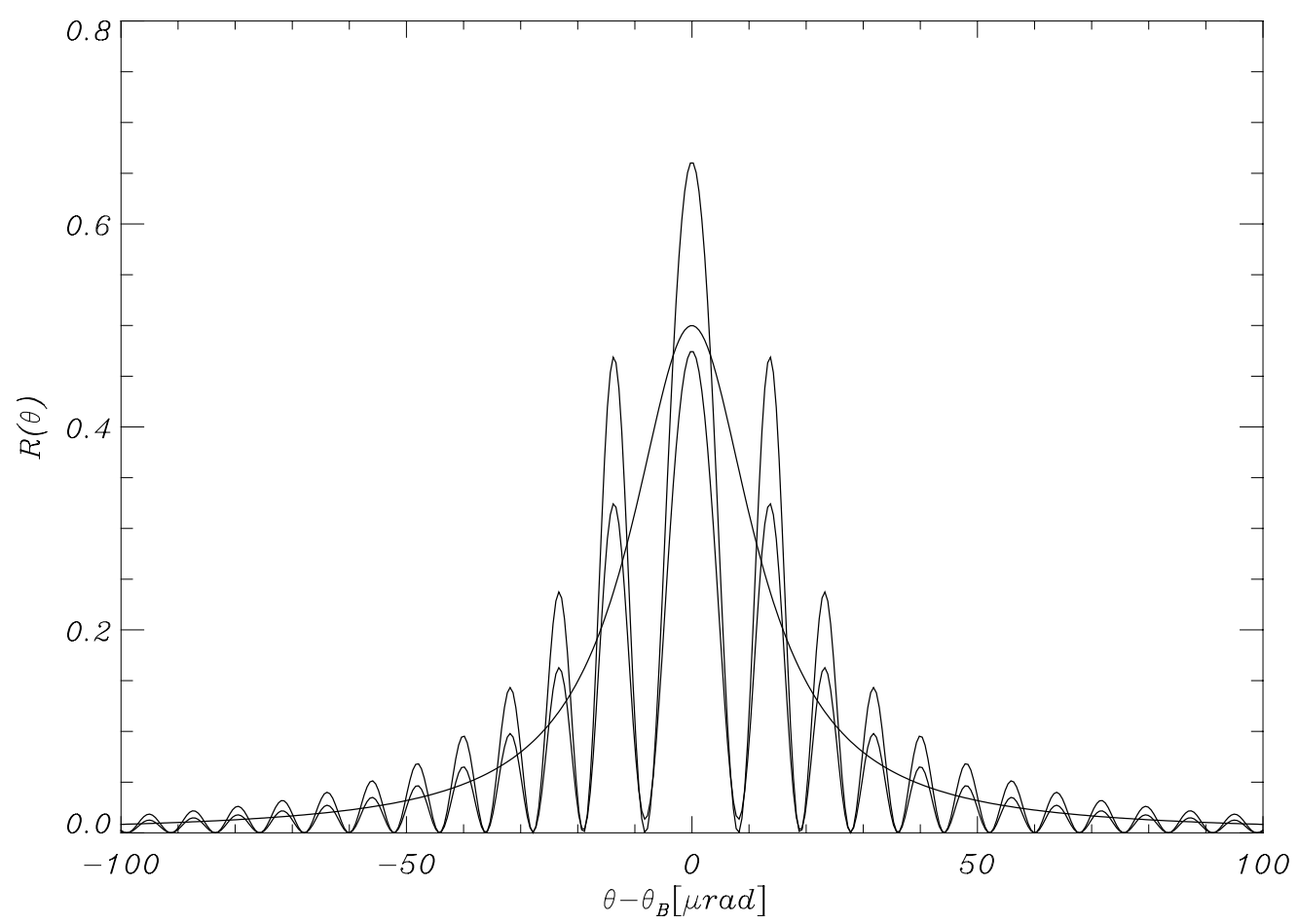

Fig 1. Laue diffraction profile for a symmetrical silicon 220 crystal at $8035 \mathrm{eV}$. The continuos line represents the diffraction curve including absorption for a $25 \mu \mathrm{m}$ thick parallel plate. The dotted line represents the same quantity but neglecting absorption. The dashed curve represents the Laue diffraction profile in the thick crystal approximation neglecting absorption. Note the Lorentzian shape of the last curve.

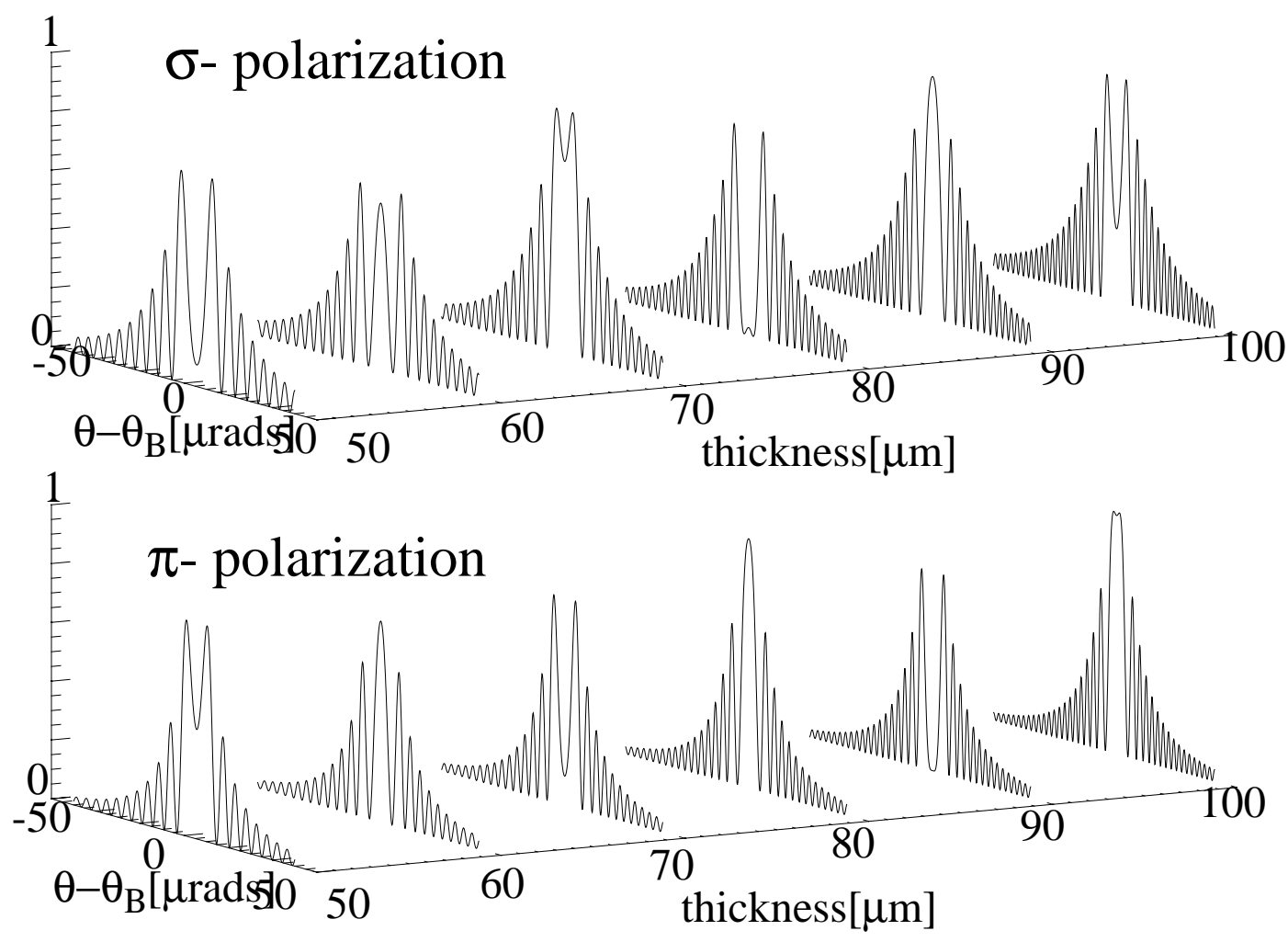

Fig. 2. Transmission Laue profiles for an asymmetrical diamond 111 crystal at 8035 $\mathrm{eV}$. Each set of curves represents one polarization case. The curves in each set are calculated for different crystal thicknesses. The asymmetry cut angle is $\alpha=70 \mathrm{deg}$, and the Bragg angle is $\theta_{\mathrm{B}}=22 \mathrm{deg}$. Note the strong dependence of the oscillations on the thickness and polarization state. 

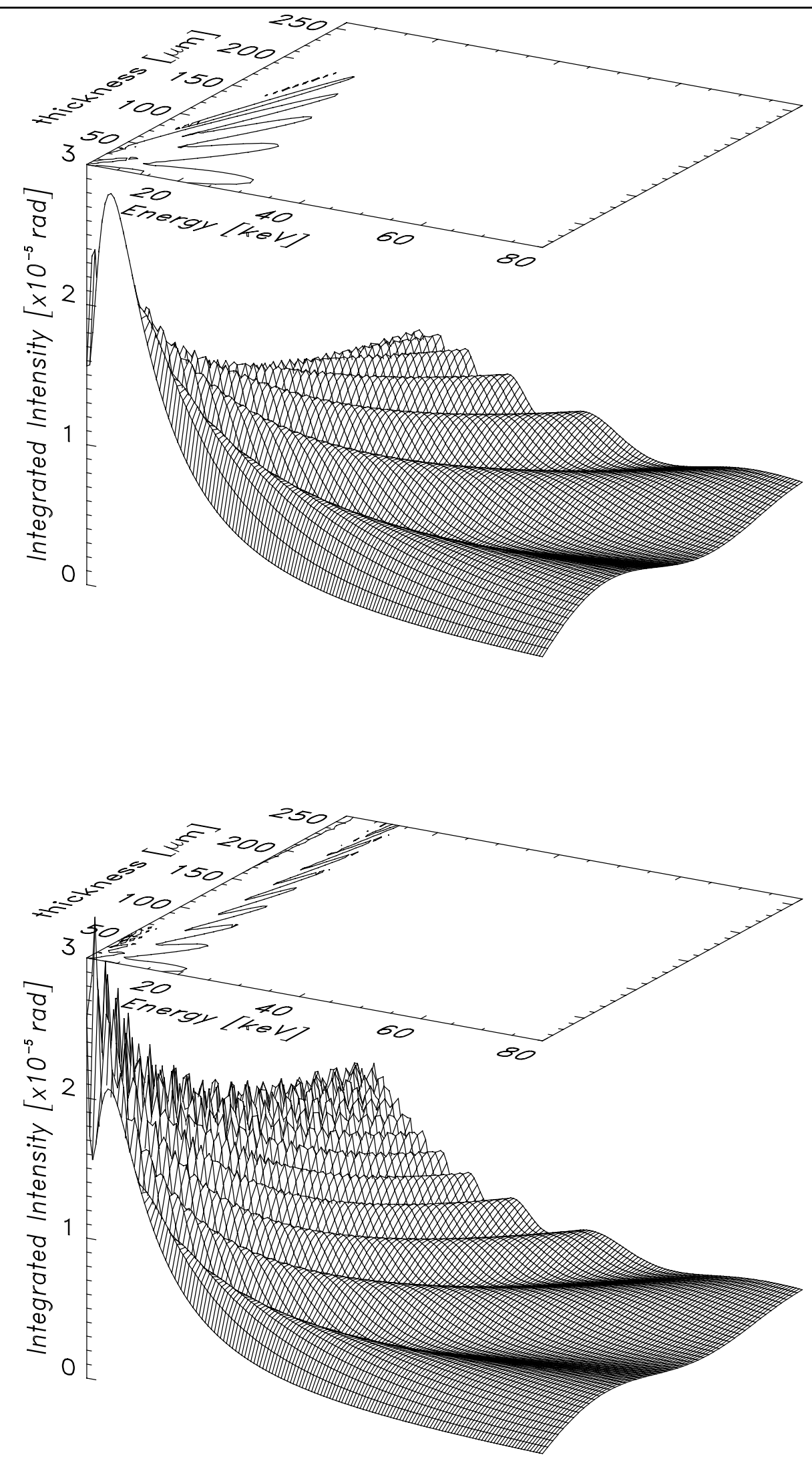

Fig. 3 Integrated intensity of Laue diffraction profiles as a function of photon energy and crystal thickness for symmetrical silicon 111 (Fig. 3a) and diamond 111 (Fig. $3 b)$ Laue crystals for $\sigma$ polarization. If one wishes to stand on a maximum of the integrated intensity for a wide energy range, then a small value of the thickness must be chosen in order to avoid jumping through different maxima. The plotted values have been calculated by numerical integration of the rocking curves produced by the BRAGG program. 


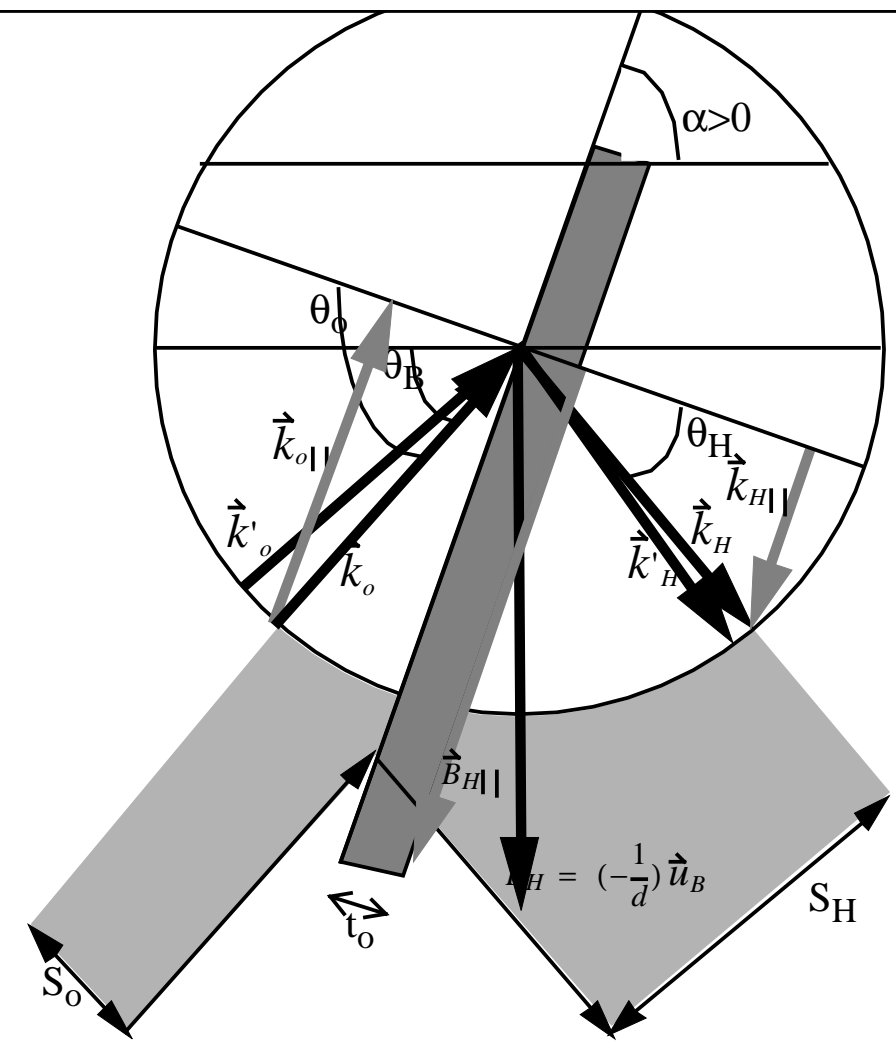

Fig. 4a. Scheme of a Laue diffraction process in a thin asymmetric crystal of asymmetry angle $\alpha$. A Laue crystal is defined as "symmetric" if $\alpha=90$ deg. Note that the output ray $\mathrm{k}_{\mathrm{H}}$ is obtained by adding the parallel components of $\mathrm{k}_{\mathrm{O}}$ and $\mathrm{B}_{\mathrm{H}}$, and conserving the $\mathrm{k}$ modulus. Another ray $\mathrm{k}_{\mathrm{o}}{ }_{\mathrm{o}}$ with the same wavelength of $\mathrm{k}_{\mathrm{O}}$ arriving on the crystal slightly away from the Bragg position is diffracted to a $\mathrm{k}^{\prime} \mathrm{H}$ using the same method. Observe that the output beam produced by a monochromatic divergent beam is still divergent.

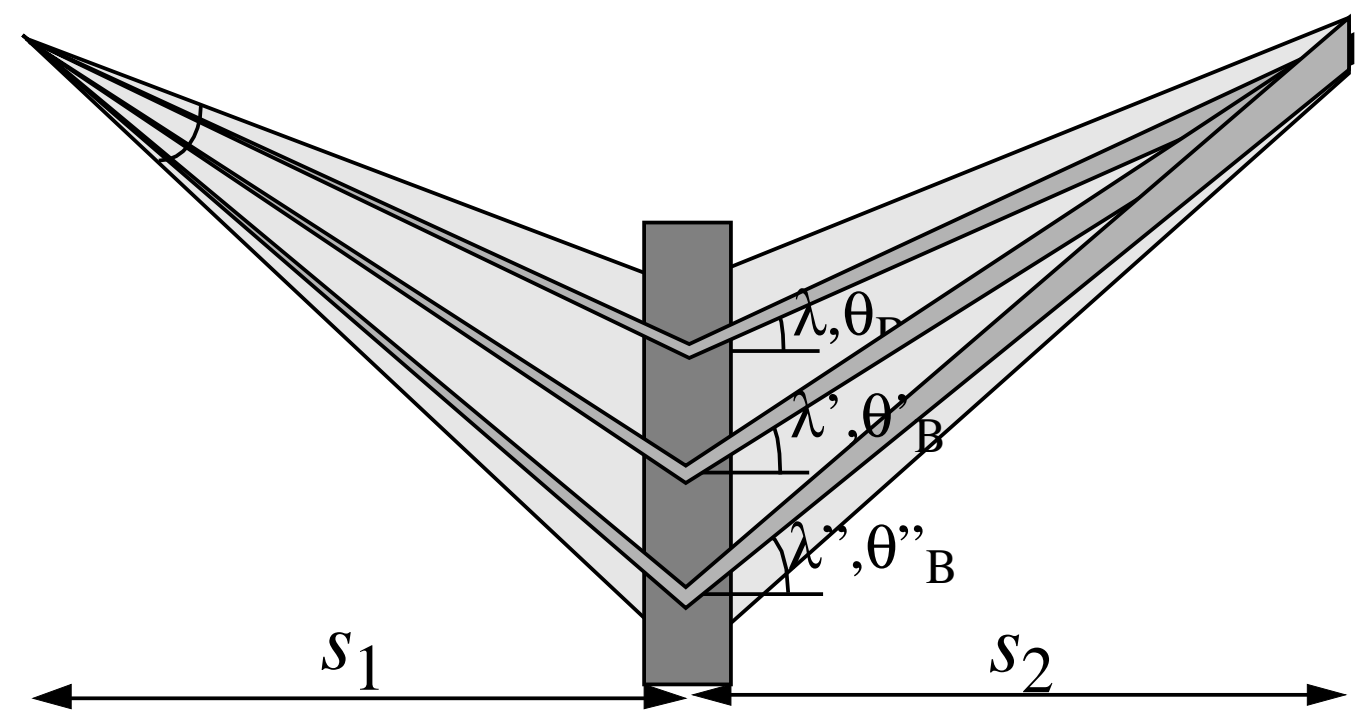

Fig $4 \mathrm{~b}$. Pseudo-focusing effect of a Laue crystal for a white incident divergent beam. The selective reflections on the Bragg planes converts the beam from divergent to convergent. This is shown for three different wavelengths. An image with the source dimension or less can never be reached in order to be consistent with the effect shown in Fig. 4a. 


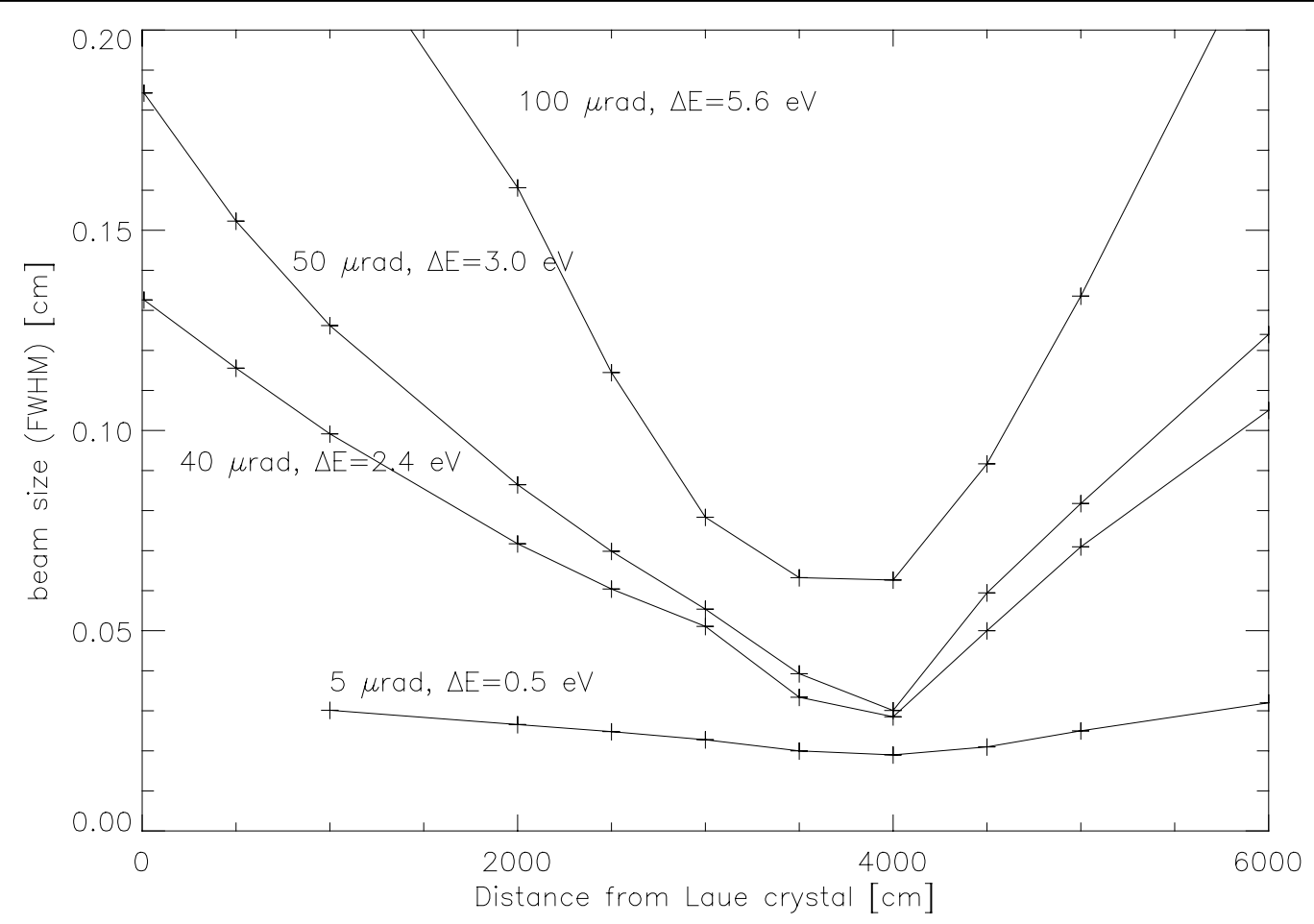

Fig. 5a. Focusing effect of a diamond 111 asymmetric Laue crystal placed at $50 \mathrm{~m}$ from a uniform divergent white source. The crystal thickness is $200 \mu \mathrm{m}$ and the asymmetry angle is $\alpha=55 \mathrm{deg}(b=1.36$ at $14 \mathrm{keV})$. The beam dimension in some points after the Laue crystal are plotted for different source divergence values. The source divergence and the crystal energy resolution are written close to their corresponding curve.

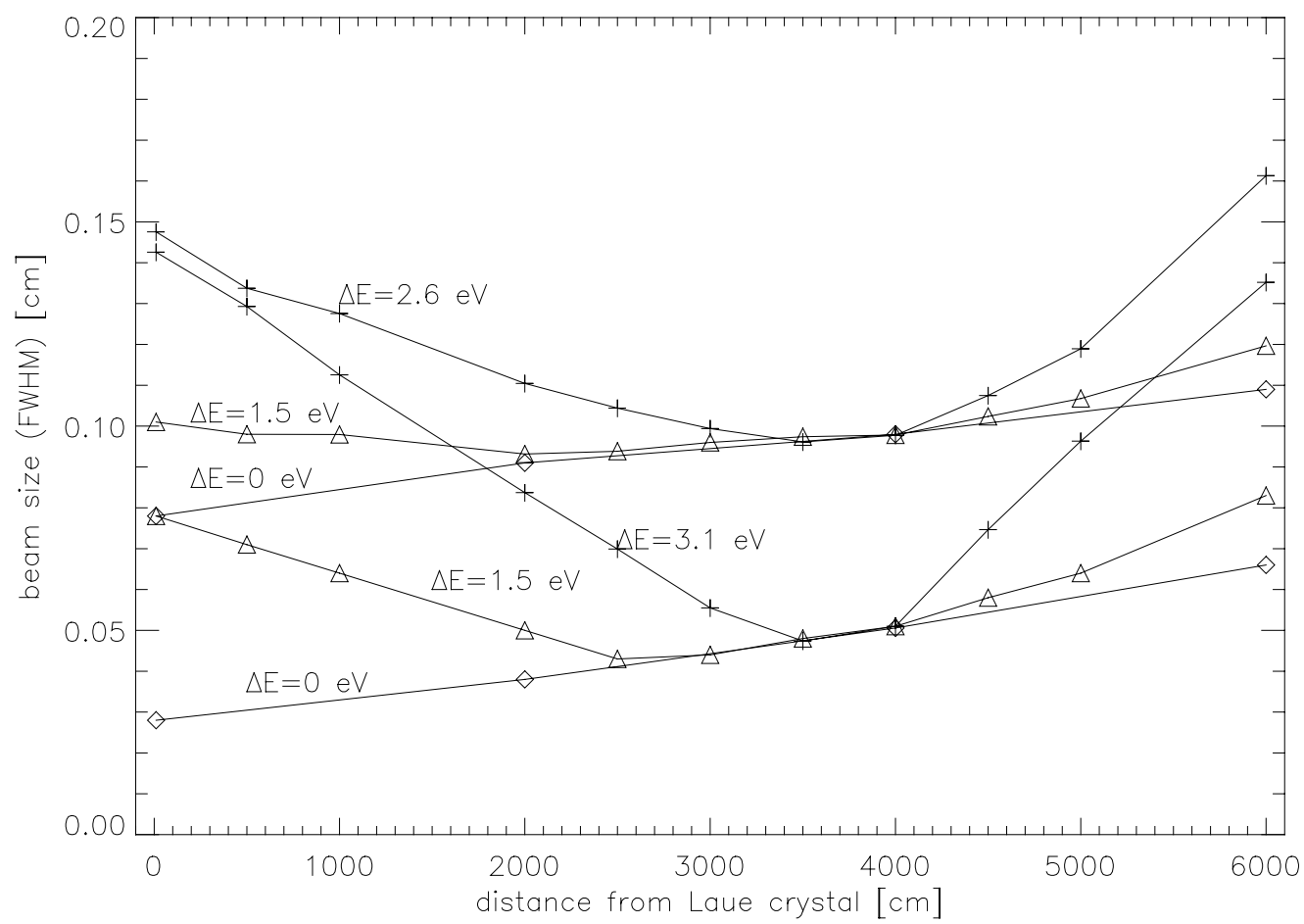

Fig. 5b. The crystal system of Fig. 5a has been analyzed with different sources. The solid line represents a gaussian divergent (fwhm $=40 \mu \mathrm{rad}$ ) and extended (fwhm $=1 \mathrm{~mm}$ ) source. The dotted lines represent a still divergent but punctual source. The "plus" and "diamond" marks refer to a polychromatic and monochromatic sources, respectively, whilst the "triangle marks" refer to a source with box energy distribution of $\Delta \mathrm{E}=1.5 \mathrm{eV}$ around $14 \mathrm{keV}$. The best focus is found at around $\mathrm{s}_{2}=\mathrm{s}_{1} / \mathrm{b}=36.6 \mathrm{~m}$ as expected (see text). 


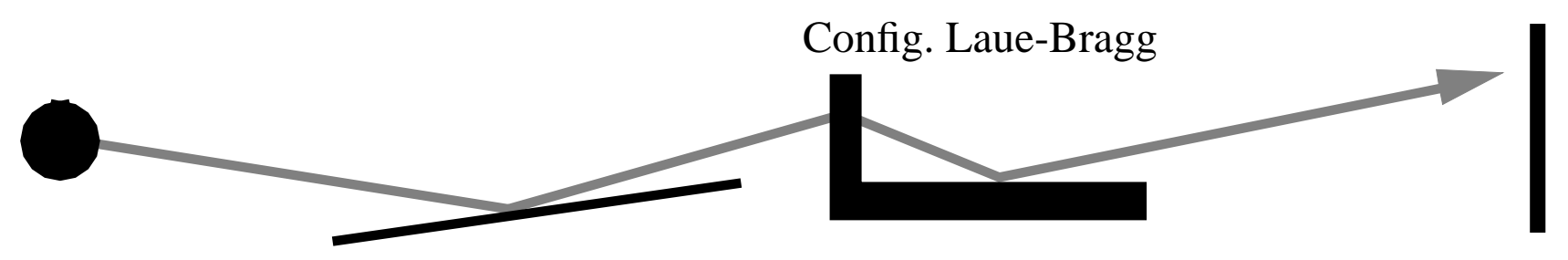

wiggler

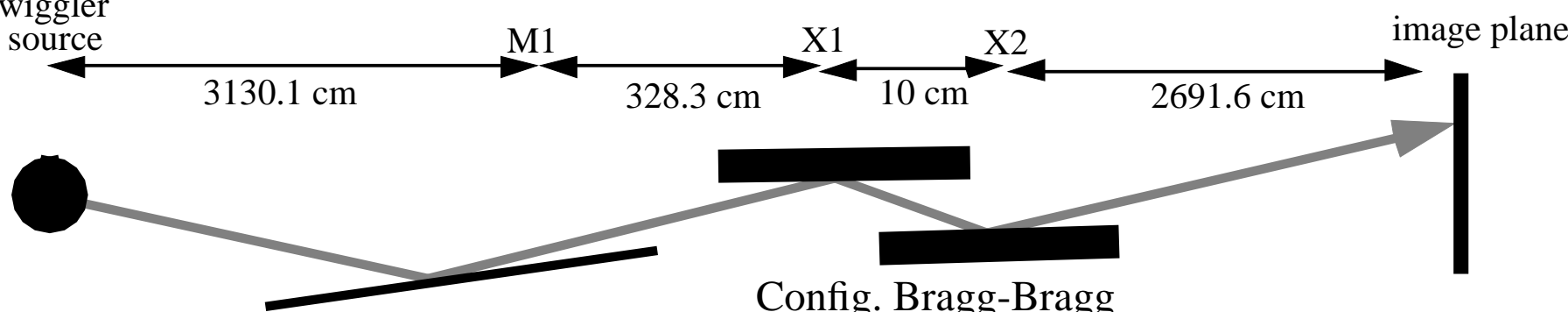

Config. Bragg-Bragg
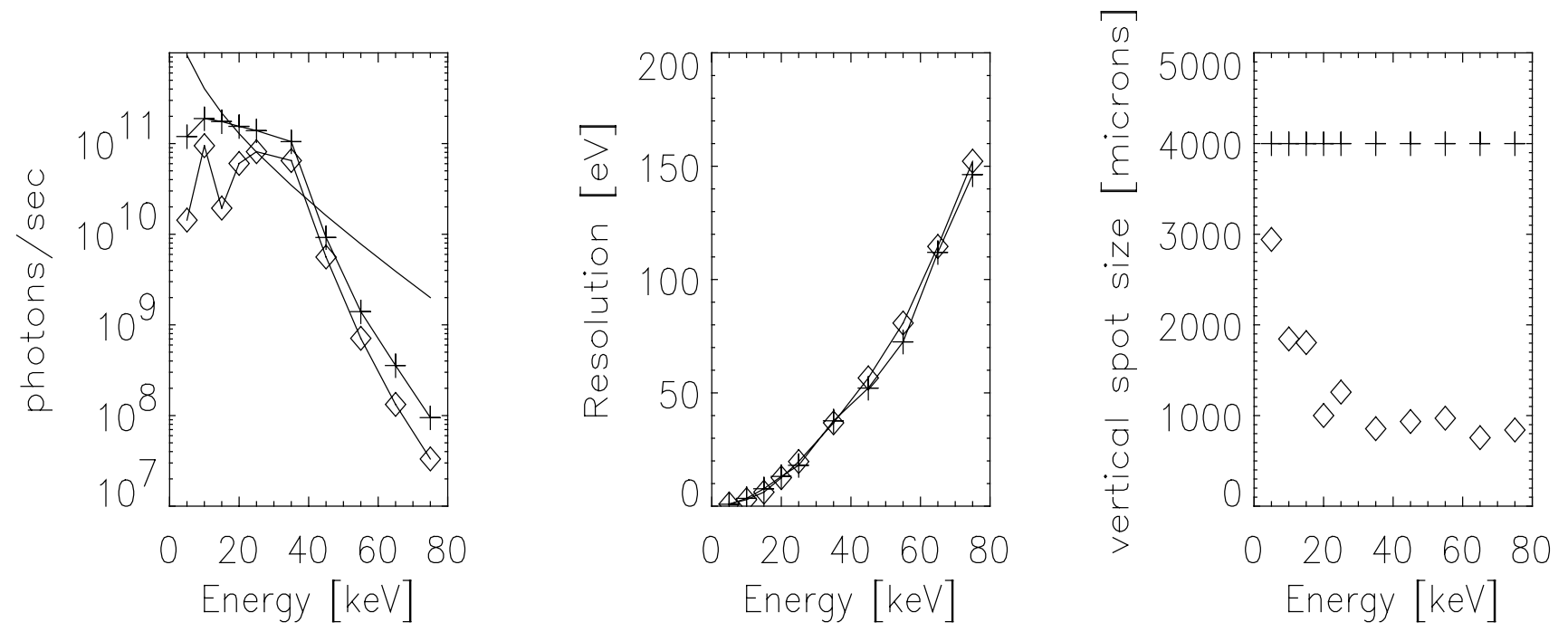

Fig. 6(top) Layout of two possible configurations for the ESRF beamline 3 [10] with the parameters considered for the ray-tracing calculations. The mirror is focusing in the sagittal direction.

Fig. 6(bottom). Photon flux (left), resolution (center) and spot size in vertical direction (right) results of the ray-tracing calculations for the system shown above. The solid curves represent the Laue-Bragg configuration whilst the dotted lines stand for the Bragg-Bragg configuration. The dashed line represent the wiggler flux in phot/s/1eV bw. The "cross" and "diamond" symbols represent the points corresponding to the calculated flux and resolution in the whole image plane for the Bragg-Bragg and Laue-Bragg configurations, respectively. The flux values are integrated in the all corresponding monochromator energy bandwidth, as indicated in the figure on the center. 